\title{
Atención de Enfermería durante el postoperatorio a paciente sometido a clipaje de aneurisma por ruptura
}

\section{Postsurgical Nursing Attention on a patient who underwent aneurysm clipping}

\section{Assistência de enfermagem no pós-operatório ao paciente submetido a ruptura de aneurisma}

\author{
J.K. Castillo-Garcia ${ }^{\mathrm{a} 1}$, E. García-Rosas ${ }^{\mathrm{b} 2 *}$, S. Cheverría-Rivera ${ }^{\mathrm{c} 2}$ \\ ORCID: \\ a $0000-0002-3748-2491$ \\ ${ }^{\mathrm{b}} 0000-0002-1786-6404$ \\ c 0000-0003-1099-8719 \\ ${ }^{1}$ Hospital General del Instituto de Seguridad y Servicios Sociales para los Trabajadores del Estado en San Luis \\ Potosí, México \\ ${ }^{2}$ Facultad de Enfermería y Nutrición, Universidad Autónoma de San Luis Potosí, San Luis Potosí, México
}

Recibido: 10 marzo 2018

Aceptado: 30 octubre 2018

\section{Resumen}

Introducción: Es reconocido que el personal de enfermería es quien tiene mayor interacción con el paciente, por lo que se ve obligado a contar con conocimientos y experiencia suficiente que permita identificar y atender las necesidades de individuos que sufren de enfermedad cerebrovascular. Esta condición de salud puede ser secundaria a la ruptura de un aneurisma, lo que conduce al paciente a un estado crítico; además, puede traducirse en riesgo para el equilibrio financiero de la familia y el Sistema Sanitario.

Métodos: Se desarrollaron las etapas del proceso de cuidado de enfermería centradas en una situación real de la práctica clínica durante el periodo postoperatorio de una paciente sometida a clipaje de aneurisma. Se utilizó el modelo de patrones funcionales de Marjory Gordon para la recolección de los datos; además de la taxonomía NANDA-NIC-NOC.

Resultados: Se presentan los planes de cuidado en los que se centró la intervención de enfermería en la paciente valorada. Así como la evaluación del proceso en las dimensiones de estructura, proceso y resultado. Discusión/conclusiones: El EVC secundario a ruptura de aneurisma cerebral es una situación grave y devastadora para la familia y el sistema sanitario. Por tanto, una adecuada atención de enfermería a este problema es de la mayor importancia. 
Palabras clave: Enfermeras clínicas; atención de enfermería; proceso de enfermería; aneurisma; enfermería perioperatoria; México.

\begin{abstract}
Introduction: It is well acknowledged that nursing staff have the greatest interaction with patients and thus, nurses need to have sufficient knowledge and experience to identify the diverse needs, for example of those patients suffering from cerebrovascular disease. This condition can be a consequence of a ruptured aneurysm, which can in turn lead the patient to a critical status jeopardizing the financial equilibrium of his/her family and the health system.

Methods: The nursing process stages were developed during the postsurgical period of a patient who underwent an aneurysm clipping. The Gordon's functional health patterns model was used to collect data, in addition to the NANDA/NIC/NOC taxonomy.

Results: Care plans derived from the nursing intervention were presented, as well as the related assessment in terms of the structure, process, and results dimensions.

Discussion/Conclusion: The CVE secondary to the rupture of an aneurysm is a serious and devastating situation for the patient, his/her family, and the health system, therefore, an adequate related nursing attention becomes of the most importance.
\end{abstract}

Keywords: Nurse Clinicians; nursing care; nursing process; aneurysm; perioperative nursing; Mexico.

\title{
Resumo
}

Introdução: Reconhece-se que os enfermeiros têm maior interação com o paciente, sendo obrigados a ter conhecimento e experiência suficientes para identificar e atender às necessidades dos indivíduos portadores de doença cerebrovascular. Essa condição de saúde pode ser secundária à ruptura de um aneurisma, que leva o paciente a um estado crítico; além disso, pode ser traduzido em risco para o equilíbrio financeiro da família e do Sistema de Saúde.

Métodos: Desenvolveram-se as etapas do processo de cuidar em enfermagem, enfocando uma situação real da prática clínica no pós-operatório de um paciente submetido a clipagem de aneurisma. O modelo de padrões funcionais de Marjory Gordon foi utilizado para coleta de dados; além da taxonomia NANDA-NIC-NOC.

Resultados: São apresentados planos de cuidados em que a intervenção de enfermagem foi focada no paciente valorizado. Assim como a avaliação do processo nas dimensões de estrutura, processo e resultado. Discussão / conclusões: A EVC secundária a um aneurisma cerebral roto é uma situação grave e devastadora para a família e o sistema de saúde. Portanto, cuidados de enfermagem adequados para este problema são de extrema importância.

Palavras-chave: Enfermeiras clínicas; cuidado de enfermagem; processo de enfermagem; aneurisma; enfermagem perioperatória; México.

\section{Introducción}

La enfermedad cerebrovascular (EVC) es considerada un problema de salud pública ${ }^{1}$ ya que, en México, es la tercera causa de mortalidad con más de 30,000 defunciones anuales; es el primer lugar en invalidez y segunda causa de muerte en los mayores de 65 años $^{2-4}$. La incidencia se incrementa paulatinamente a partir de los 55 años de edad ${ }^{5}$. Además, de contribuir con cerca de la mitad de los problemas neurológicos atendidos en los hospitales generales ${ }^{6}$. En 2014, la mortalidad asociada a EVC en México fue de $33,144^{7}$.

Esta patología es causada por la lesión de la pared del vaso sanguíneo, la ruptura, la oclusión por émbolos y alteraciones de la viscosidad de la sangre ${ }^{8,9}$. Se clasifica en isquémicas y hemorrágicas ${ }^{10}$. De las últimas, entre el 22 al 29\% son por rupturas de un aneurisma cerebral, donde la mayoría de los pacientes 
mueren en las primeras 24 horas por falta de atención médica; y entre el $40 \%$ al $60 \%$ en los primeros 30 días como consecuencia de complicaciones ${ }^{11}$.

El tratamiento más común para el EVC secundario a ruptura de aneurisma es la colocación de clips metálicos en el cuello del aneurisma, con técnica microquirúrgica a fin de lograr su oclusión ${ }^{\text {. Cerca de }}$ un tercio de estos pacientes recupera su independencia funcional, la gran mayoría egresa del hospital con discapacidad y presentan déficit neurológico, lo que afecta negativamente su calidad de vida ${ }^{12}$. Por ello, es de gran importancia los cuidados de enfermería de manera temprana, sumados a rehabilitación y la enseñanza a la familia sobre los cuidados de estos pacientes ${ }^{13}$.

Los pacientes que sufren de esta patología se ubican en servicios donde se atienden problemas de salud de alta complejidad. Es el personal de enfermería quien pasa mayor parte en contacto directo con estos pacientes. Por esto, es necesario que este personal cuente con los conocimientos y experiencia necesaria, para atender las necesidades asistenciales ${ }^{14}$.

El personal de enfermería debe estar consciente de sus conocimientos, deberes y principios teóricos, ya que esto le permitirá planificar y ejecutar los cuidados orientados, para atender a los pacientes procedentes de cirugía con complejidades variadas y con necesidades de cuidados especializados específicos ${ }^{15}$.

El personal de enfermería, como en otras instituciones y áreas utiliza el Proceso de Cuidado de Enfermería (PCE), para brindar atención a los pacientes, método específico de la profesión caracterizado por ser sistemático, organizado, cíclico y dinámico; fundamento de toda intervención al brindar el cuidado ${ }^{16}$ y herramienta fundamental de la práctica asistencial de la enfermería ${ }^{17}$.

El PCE tiene cinco etapas: 1) valoración (donde se realiza la recolección de información de diversas fuentes); 2) diagnóstico, que implica la realización de un juicio clínico en función con la respuesta humana ${ }^{18}$; 3) planificación, es el momento en que se determinan las intervenciones y actividades específicas; 4) ejecución, donde se lleva a la práctica el plan de cuidado; 5) evaluación, es la etapa donde se determinan la eficacia de las intervenciones y la consecución de resultados ${ }^{19}$.

Durante el postoperatorio, la atención se centra en torno a la valoración integral del paciente y su vigilancia continua, a fin de identificar signos y síntomas manifestados, con el fin de anticiparse a prevenir complicaciones. El objetivo de los cuidados durante esta fase, es contribuir a recuperar el equilibrio fisiológico, con el mínimo de complicaciones, con la mayor calidad en la atención prestada. Aquí radica la importancia de la labor de los profesionales de enfermería.

El interés de este trabajo es mostrar el impacto de los cuidados postquirúrgicos y ofrecer un conocimiento argumentado en el PCE centrado en un caso real. El caso se ubica en una unidad de tercer nivel de atención en México.

\section{Metodología}

Para el presente caso, se buscaron antecedentes de dos fuentes: el expediente clínico, y mediante una entrevista al cuidador primario (hija). La recolección de datos se realizó bajo el modelo de la Teoría de los Patrones Funcionales de Marjory Gordon, como se sugiere en el libro de diagnósticos de enfermería de la Asociación Norteamericana de Diagnósticos de Enfermería. Al cuidador primario se le informó la finalidad de la entrevista, así como la confidencialidad de los datos recabados, los beneficios y que no comprometía ninguna situación que pusiera en riesgo la salud de su familiar, posterior a ello, firmó la carta de consentimiento informado. Se tomó en cuenta la declaración de Helsinki de 1975, enmendada en 1989. A ello, se sumaron los lineamientos del Reglamento de la Ley General de Salud en Materia de Investigación para la Salud en los seres humanos.

\section{Antecedentes}

Se trata de paciente femenino de 68 años de edad, con diabetes e hipertensión, que acude el día 08 de agosto al servicio de urgencias en el Hospital General por presentar cefalea intensa holocraneana con EVA 8/10, resto sin importancia. Se brindó apoyo con analgésicos antiinflamatorios no esteroideos y fue 
enviada a domicilio. Dos días después, el día 10, es llevada por sus familiares por presentar cefalea, náuseas y vértigo, así como disminución de movilidad en las extremidades superiores e inferiores izquierdas, con cifras de presión arterial de 190/120mmHg; se sospecha de evento vascular cerebral por lo que se refiere al tercer nivel de atención, por falta de recursos en la primera institución. El día 11 del mismo mes es enviada a una unidad de tercer nivel, donde a su ingreso se le realizó TAC de cráneo, angiotac y angiografía, con evidencia de hemorragia subaracnoidea Fisher II + aneurisma sacular en arteria cerebral media parasagital derecha de $8 \mathrm{~mm}$. Se programa para clipaje de aneurisma el día 13. Se realiza valoración en el área de hospitalización el día 22 agosto.

\section{Valoración de enfermería por patrones funcionales de Marjory Gordon}

Datos generales

Paciente: HTMG Edad: 68 años

Diagnóstico Médico: Hipertensión arterial sistémica, hemorragia subaracnoidea Fisher II + aneurisma sacular en la arteria cerebral media + post-operada de clipaje de aneurisma.

\section{Patrón Percepción-Manejo de la salud}

Interrogatorio indirecto (hija): familiar refiere que la paciente radica en la Ciudad de México, se dedica a las labores del hogar; habita en casa propia con los servicios básicos de la vivienda. Esquema de vacunación desconocido. Hipertensión de 10 años de evolución, bajo tratamiento con nifedipino comprimidos de liberación prolongada 10mg; deficiente apego al tratamiento farmacológico. Hace 25 años se realizó oclusión tubárica bilateral. Niega toxicomanías y alérgicos. El familiar refiere que en general su estado de salud había sido bueno. Se observa paciente en malas condiciones generales, con higiene deficiente. En el área de hospitalización tiene tratamiento farmacológico con fenitoína $125 \mathrm{mg}$ IV c/12h.; omeprazol 40 mg IV c/24h.; ketoprofeno 100 mg IV c/8h., paracetamol 1 gr IV c/8h., clindamicina $600 \mathrm{mg}$ IV c/12h., cefatriaxona 1 gr IV c/12h., desxametasona 8 mg IV c/12h. Precedex $200 \mathrm{mcg}$ IV en infusión, fentanilo $1 \mathrm{mg}+230 \mathrm{ml}$ solución glucosada $5 \%$ a $10 \mathrm{ml} / \mathrm{h}$, soluciones parenterales de 1000 $\mathrm{ml}$ de solución fisiológica a $40 \mathrm{ml} / \mathrm{h}$.

\section{Patrón Nutricional-Metabólico}

Se encuentra paciente con temperatura de $36^{\circ} \mathrm{C}$, IMC de 27.5. Piel con disminución de la turgencia, palidez de tegumentos. Catéter Venoso Central de dos lúmenes subclavio derecho. Cráneo normocefálico con presencia de herida quirúrgica temporoparietal de lado derecho, sin datos de infección, rigidez de la nuca y poco sostén con desviación hacia la izquierda. Cabello bien implantado. Mucosa oral semihidratada, labios secos, ausencia parcial de dientes, sialorrea, cierre incompleto de la boca. Extremidades sin edema con presencia de medias compresivas. Llenado capilar de 4", con apoyo de nutrición enteral por sonda de gastrostomía. Laboratorios: Hb $10.8 \mathrm{~g} / \mathrm{dl}$, HCT 34.8\%, plaquetas 417 000, TP $13.3 \mathrm{~s}$, TPT 30.6 s. INR 1.0, glucosa $105 \mathrm{mg} / \mathrm{dl}$, urea $30 \mathrm{mg} / \mathrm{dl}$, creatinina $0.34 \mathrm{mg} / \mathrm{dl}$, Na $142 \mathrm{mMol} / \mathrm{L}, \mathrm{K} 3.41$ $\mathrm{mMol} / \mathrm{L}, \mathrm{Cl} 109.1 \mathrm{mMol} / \mathrm{L}$.

\section{Patrón Eliminación}

Paciente con sonda vesical tipo Foley \#16 Fr, con volumen urinario de $1320 \mathrm{ml}$ en $7 \mathrm{~h}$., turno, con densidad de 1010. Uresis con características normales. Evacuaciones semilíquidas, color café, fétidas. Ruidos peristálticos de 6 $\mathrm{x}^{\prime}$. Abdomen blando, depresible, sin distensión.

\section{Patrón Actividad-Ejercicio}

Frecuencia cardiaca de $90 x^{\prime}$ respiratoria de $24 x^{\prime}$, tensión arterial de 140/75 mmHg, saturación de $91 \%$. Traqueostomía con apoyo de $\mathrm{O}_{2}$ por nebulizador al $60 \%$ con pieza en “ $\mathrm{T}$ ”. La exploración física revela tórax simétrico, normoexpandible, campos pulmonares con estertores en bases, sin masas palpables. 
Ruidos cardiacos rítmicos, fuertes y audibles. Resto sin compromiso. No se cuenta con gasometría arterial del día.

Patrón Sueño-Descanso

No valorable.

\section{Patrón Cognitivo-Perceptual}

Responde a reflejos dolorosos y estímulos verbales' (Glasgow 10 pts), se observan gesticulaciones de dolor.

Reflejos: lado derecho e izquierdo

a) Bicipital: normorreflexia, hiporreflexia

b) Rotuliano: normorreflexia, izquierdo hiporreflexia

c) Aquiliano: normorreflexia, hiporreflexia

\begin{tabular}{ll} 
Par Craneal & Resultado \\
\hline I.- Nervio Olfativo & No valorable \\
\hline II.- Nervio Óptico & $\begin{array}{l}\text { Fundoscopia no pudo realizarse por miosis } \\
\text { y ptosis palpebral izquierda }\end{array}$ \\
\hline $\begin{array}{l}\text { III.- Nervio Oculomotor/Motor Ocular Común } \\
\text { IV.- Nervio Troclear/Patético } \\
\text { VI.- Nervio Abductor/Ocular Externo }\end{array}$ & $\begin{array}{l}\text { Mirada primaria central, pupilas mióticas. } \\
\text { Reflejos fotomotor y consensual bilaterales }\end{array}$ \\
\hline V.- Nervio Trigémino & Reflejo corneal \\
\hline VII.- Nervio Facial & Gesticulación disminuida \\
\hline VIII.-Nervio Vestibuloclear/Auditivo & Presente \\
\hline IX.- Nervio Glosofaríngeo & Reflejo tusígeno disminuido \\
X.- Nervio Vago/Neumogástrico & No valorable \\
\hline XI.- Nervio Accesorio & Reflejo de deglución disminuido \\
\hline XII.- Nervio Hipogloso &
\end{tabular}

\section{Patrón Autopercepción-Autoconcepto}

No valorable.

\section{Patrón Rol-Relaciones}

Vive con su hija y un nieto, es divorciada, ama de casa. (Fuente secundaria).

\section{Patrón Sexualidad-Reproducción}

Gestas 5, Partos 5, Abortos 0, Cesárea 0, IVSA: se desconoce. Integridad de genitales. A la exploración física se encuentra integridad de genitales.

Patrón Adaptación-Tolerancia al estrés

No valorable.

Patrón Valores-Creencias

Religión católica. 


\begin{tabular}{|c|c|c|}
\hline Racimos & Diagnóstico & Prioridad \\
\hline $\begin{array}{l}\text { Presencia de secreciones. } \\
\text { Estertores en bases. } \\
\text { Disnea. } \\
\text { Presencia de secreciones en boca. } \\
\text { Taquipnea. }\end{array}$ & $\begin{array}{l}\text { Limpieza ineficaz de la vía aérea } \mathrm{r} / \mathrm{c} \text { vía } \\
\text { aérea artificial } \mathrm{m} / \mathrm{p} \text { sonidos respiratorios } \\
\text { adventicios, excesiva cantidad de esputo, } \\
\text { cambios en la frecuencia respiratoria. }\end{array}$ & \multirow{4}{*}{$\begin{array}{l}\text { Prioridad 1. Problemas } \\
\text { que representan una } \\
\text { amenaza para las } \\
\text { necesidades fisiológicas } \\
\text { (problemas respiratorios, } \\
\text { circulación, nutrición, } \\
\text { hidratación, regulación } \\
\text { de la temperatura). }\end{array}$} \\
\hline $\begin{array}{l}\text { Presencia de secreciones en boca. } \\
\text { Mantiene la boca abierta disminución de la } \\
\text { función del nervio glosofaríngeo e hipogloso. } \\
\text { Alimentación asistida por } \\
\text { medio de gastrostomía. }\end{array}$ & $\begin{array}{l}\text { Deterioro de la deglución r/c deterioro } \\
\text { neuromuscular m/p sialorrea, cierre incompleto } \\
\text { de los labios y dificultad en la deglución. }\end{array}$ & \\
\hline $\begin{array}{l}\text { Hemiparesia izquierda. } \\
\text { No se mantiene en posición al movilizarla. }\end{array}$ & $\begin{array}{l}\text { Deterioro de la movilidad física r/c } \\
\text { deterioro neuromuscular m/p inestabilidad } \\
\text { postural, limitación de la capacidad para } \\
\text { las habilidades motoras finas y gruesas. }\end{array}$ & \\
\hline $\begin{array}{l}\text { Hemiplejia de lado izquierdo. } \\
\text { Poco sostén. } \\
\text { Desviación a la izquierda. }\end{array}$ & $\begin{array}{l}\text { Desatención unilateral r/c lesión cerebral } \\
\text { causada por problemas vasculares cerebrales m/p } \\
\text { marcada desviación de la cabeza hacia el lado no } \\
\text { desatendido ante estímulos, no tener conciencia } \\
\text { de posición de la extremidad desatendida. }\end{array}$ & \\
\hline $\begin{array}{l}\text { Herida quirúrgica en área } \\
\text { fronto-temporal derecha. } \\
\text { Presencia de CVC. } \\
\text { Traqueostomía. }\end{array}$ & $\begin{array}{l}\text { Riesgo de infección } \mathrm{r} / \mathrm{c} \text { defensas } \\
\text { primarias inadecuadas: rotura de la } \\
\text { piel (procedimientos invasivos). }\end{array}$ & $\begin{array}{l}\text { Prioridad 2. Seguridad y } \\
\text { protección.- problemas } \\
\text { que representan } \\
\text { una amenaza para la } \\
\text { protección y seguridad. }\end{array}$ \\
\hline
\end{tabular}

\section{Plan de Intervención y Evaluación por Diagnóstico de Enfermería con base en NANDA}

Dominio 11: Seguridad / Protección

Clase 02: Lesión Física

Etiqueta: (00031) Limpieza ineficaz de las vías aéreas

Definición: Incapacidad para eliminar las secreciones u obstrucciones del tracto respiratorio para mantener las vías aéreas permeables.

Diagnóstico de Enfermería: Limpieza ineficaz de la vía aérea r/c vía aérea artificial m/p sonidos respiratorios adventicios, excesiva cantidad de esputo y cambios en la frecuencia respiratoria.

Clasificación de los resultados de Enfermería (NOC)

\begin{tabular}{|c|c|c|c|c|}
\hline \multirow[t]{2}{*}{ Resultado } & \multirow[t]{2}{*}{ Indicadores } & \multirow[t]{2}{*}{ Escala de medición } & \multicolumn{2}{|c|}{ Puntuación Diana } \\
\hline & & & Mantener & Aumentar \\
\hline Dominio 02: Salud & 41004 Frecuencia & 1 Gravemente comprometido o grave & 3 & 5 \\
\hline Fisiológica & respiratoria & 2 Sustancialmente comprometido o sustancial & 2 & 4 \\
\hline Clase E: & 41012 Capacidad de & 3 Moderadamente comprometido o moderado & 3 & 1 \\
\hline Cardiopulmonar & eliminar las secreciones & 4 Levemente comprometido o levente & 2 & 5 \\
\hline $\begin{array}{l}\text { Resultado } \\
\text { 0410.- Estado } \\
\text { respiratorio: } \\
\text { permeabilidad de } \\
\text { las vías respiratorias }\end{array}$ & $\begin{array}{l}41018 \text { Uso de } \\
\text { músculos accesorios } \\
41020 \text { Acumulación } \\
\text { de esputo }\end{array}$ & 5 No comprometido & $\mathrm{T}=10$ & $\mathrm{~T}=15$ \\
\hline Dominio 02: Salud & 41501 Frecuencia & 1 Desviación grave del rango normal & 4 & 4 \\
\hline Fisiológica & Respiratoria & 2 Desviación sustancial del rango normal & 3 & 5 \\
\hline Clase E: & 41502 Ritmo Respiratorio & 3 Desviación moderada del rango normal & 3 & 5 \\
\hline Cardiopulmonar & 41504 Ruidos & 4 Desviación leve del rango normal & 4 & 5 \\
\hline Resultado & respiratorios auscultados & 5 Sin desviación del rango normal & 2 & 4 \\
\hline 0415: Estado & 41508 Saturación & & $\mathrm{T}=16$ & $\mathrm{~T}=23$ \\
\hline \multirow[t]{3}{*}{ Respiratorio } & de oxígeno & & & \\
\hline & 41522 Sonidos & & & \\
\hline & respiratorios adventicios & & & \\
\hline
\end{tabular}


Intervención: 3160 Aspiración de las vías aéreas

Campo 02.- Fisiológico complejo

Clase I.- Control neurológico

Actividades:

- Determinar la necesidad de aspiración oral y/o traqueal.

- Auscultar los sonidos respiratorios antes y después de la aspiración.

- Informar al paciente y a la familia sobre la aspiración.

- Usar precauciones universales.

- Utilizar equipo desechable estéril para cada procedimiento de aspiración traqueal.

- Monitorizar el estado de oxigenación del paciente (niveles de $\mathrm{SaO} 2$ ), estado neurológico, estado hemodinámico (nivel de PAM y ritmo cardíaco) inmediatamente antes, durante y después a la aspiración.

- Limpiar la zona alrededor de la estoma traqueal después de terminar la aspiración.

- Detener la aspiración traqueal y suministrar oxígeno suplementario si el paciente experimenta bradicardia, un aumento de las extrasístoles ventriculares y/o desaturación.

- Controlar y observar el color, cantidad y consistencia de las secreciones.
Fundamentación:

- La afección de los pares craneales bajos pueden repercutir en la capacidad de deglución por disminución de la función faríngea lo cual provoca acumulación de residuos y debilidad de los músculos. La prevalencia de la aspiración después de sufrir un ACV es del 25\% al 30\%.

- La valoración precisa, evita riesgos y mayor compromiso respiratorio del paciente.

- La auscultación de campos pulmonares permite evaluar la eficacia del procedimiento.

- Una información adecuada reduce al mínimo la angustia y el temor del paciente.

- Limitar el tiempo de aspiración ayuda a evitar la hipercapnia lo que puede aumentar la vasodilatación cerebral y así evitar la hipoxia que puede aumentar la isquemia cerebral.

- La pulsioxímetria ofrece datos continuos de la saturación de oxígeno.

- Un registro de las características de las secreciones permite identificar complicaciones infecciosas del árbol bronquial.

- Las secreciones viscosas son difíciles de expectorar y pueden ocasionar tapones de moco que a su vez pueden provocar atelectasias.

Intervención: 3140 Manejo de la vía aérea

Campo 02.- Fisiológico complejo

Clase I.- Control neurológico

\begin{tabular}{ll}
\hline Actividades: & Fundamentación: \\
- Eliminar las secreciones fomentando & - La tos ayuda a movilizar, desprender y reducir las secreciones \\
la tos o mediante succión. & y por lo tanto disminuye la intensidad de aspiración. \\
- Auscultar los sonidos respiratorios, observando las & • Los cambios en la respiración y en los ruidos de percusión \\
áreas de disminución o ausencia de ventilación & denotan un aumento en la densidad del tejido pulmonar como \\
y la presencia de sonidos adventicios. & consecuencia de una acumulación de secreciones o líquidos, \\
-Vigilar el estado respiratorio y de oxigenación. & provoca que los bronquios se dilaten, por la tanto hay una \\
& disminución en la resistencia aérea lo cual permite el flujo de aire.
\end{tabular}

Intervención: 3320 Oxigenoterapia

Campo 02.- Fisiológico complejo

Clase I.- Control neurológico

Actividades:

- Eliminar las secreciones traqueales.

- Mantener la permeabilidad de las vías aéreas.

- Verificar el flujo de litros de oxígeno.

- Comprobar periódicamente el dispositivo de aporte de oxígeno para asegurar que se administra la concentración prescrita.

- Controlar la eficacia de la oxigenoterapia (pulsioxímetro, gasometría arterial), según corresponda.
Fundamentación:

- Mantener libre de secreciones la vía respiratoria en pacientes inconscientes, intubados o debilitados.

- Los conocimientos del personal de enfermería sobre el funcionamiento del equipo de oxígeno, el abastecimiento suficiente de este gas, un regulador que garantice el flujo en cantidad fija y continua, un aparato en buenas condiciones de uso, son requerimientos básicos para su eficaz administración.

\section{Evaluación de proceso}

Durante el desarrollo de las actividades de enfermería señaladas en el apartado anterior, no se presentaron situaciones adversas que comprometieran la salud del paciente y que limitaran la realización de las mismas; las actividades se realizaron en su totalidad. 


\section{Evaluación de Estructura}

El personal de enfermería contó con las competencias necesarias para la realización de las actividades desarrolladas; así como los recursos materiales para la realización de las mismas.

\section{Evaluación de resultado}

La paciente mostró evidencia de que las intervenciones de enfermería fueron efectivas; frecuencia respiratoria dentro de parámetros normales, campos pulmonares limpios y bien ventilados, coloración de tegumentos normal, saturación de oxígeno de 97\%.

\section{Plan de Cuidados de Enfermería}

Dominio 02: Nutrición

Clase 01: Ingestión

Etiqueta: (00103) Deterioro de la deglución

Definición: Funcionamiento anormal del mecanismo de la deglución asociado con déficit de la estructura o función oral, faríngea o esofágica.

Diagnóstico de Enfermería: Deterioro de la deglución r/c deterioro neuromuscular m/p sialorrea, cierre incompleto de los labios y dificultad en la deglución.

Clasificación de los Resultados de Enfermería (NOC)

\begin{tabular}{|c|c|c|c|c|}
\hline \multirow[t]{2}{*}{ Resultado } & \multirow[t]{2}{*}{ Indicadores } & \multirow[t]{2}{*}{ Escala de Medición } & \multicolumn{2}{|c|}{ Puntuación Diana } \\
\hline & & & Mantener & Aumentar \\
\hline $\begin{array}{l}\text { Dominio 02: Salud Fisiológica } \\
\text { Clase J: Neurocognitiva } \\
\text { Resultado }\end{array}$ & 91306 Sensibilidad facial & $\begin{array}{l}1 \text { Gravemente } \\
\text { comprometido o grave }\end{array}$ & 3 & 4 \\
\hline \multirow[t]{4}{*}{$\begin{array}{l}\text { 913.- Estado Neurológico: Función } \\
\text { sensitiva/motora de pares craneales }\end{array}$} & $\begin{array}{l}91307 \text { Movimiento } \\
\text { muscular facial }\end{array}$ & $\begin{array}{l}2 \text { Sustancialmente } \\
\text { comprometido o sustancial }\end{array}$ & 4 & 5 \\
\hline & 91308 Deglución & $\begin{array}{l}3 \text { Moderadamente } \\
\text { comprometido o moderado }\end{array}$ & 3 & 4 \\
\hline & 91310 Movimiento lingual & $\begin{array}{l}4 \text { Levemente } \\
\text { comprometido o levente }\end{array}$ & 3 & 4 \\
\hline & $\begin{array}{l}91326 \text { Parálisis } \\
\text { facial unilateral }\end{array}$ & 5 No comprometido & $\begin{array}{c}2 \\
\mathrm{~T}=15\end{array}$ & $\begin{array}{c}4 \\
\mathrm{~T}=15\end{array}$ \\
\hline $\begin{array}{l}\text { Dominio 02: Salud Fisiológica } \\
\text { Clase K: Digestión y nutrición }\end{array}$ & 101003 Producción de saliva & & 3 & 4 \\
\hline $\begin{array}{l}\text { Resultado } \\
\text { 1010: Estado de deglución } \\
\text { Dominio 02: Salud Fisiológica }\end{array}$ & $\begin{array}{l}101004 \text { Capacidad } \\
\text { de masticación }\end{array}$ & & 2 & 4 \\
\hline \multirow{5}{*}{$\begin{array}{l}\text { Clase K: Digestión y nutrición } \\
\text { Resultado } \\
\text { 1015.- Función gastrointestinal }\end{array}$} & $\begin{array}{l}101010 \text { Momento del } \\
\text { reflejo de deglución }\end{array}$ & & $\begin{array}{c}2 \\
\mathrm{~T}=7\end{array}$ & $\begin{array}{c}4 \\
T=12\end{array}$ \\
\hline & $\begin{array}{l}101501 \text { Tolerancia a } \\
\text { la alimentación }\end{array}$ & & 3 & 4 \\
\hline & $\begin{array}{l}101503 \text { Frecuencia } \\
\text { de deposiciones }\end{array}$ & & 3 & 4 \\
\hline & 101508 Ruidos abdominales & & 3 & 4 \\
\hline & 101537 Pérdida de peso & & $\begin{array}{c}3 \\
\mathrm{~T}=12\end{array}$ & $\begin{array}{c}5 \\
\mathrm{~T}=17\end{array}$ \\
\hline
\end{tabular}


Intervención: 2620 Monitorización neurológica

Campo 02.- Fisiológico complejo

Clase I.- Control neurológico

\begin{tabular}{ll}
\hline Actividades: & Fundamentación: \\
- Comprobar el tamaño, forma, simetría y & • Cualquier cambio en la pupila indica presión \\
capacidad de la reacción de las pupilas. & en el III par craneal o en el nervio óptico. \\
- Vigilar el nivel de conciencia. & - Determinar precozmente signos y síntomas de hipertensión \\
- Monitorizar los signos vitales. & intracraneal como bradicardia, hipertensión arterial \\
- Evitar actividades que aumenten la PIC. & y alteraciones respiratorias (Triada de Cushing). \\
& La alteración del nivel de conciencia es \\
& secundario a la disminución de la presión de \\
& perfusión cerebral y disminución del FSC.
\end{tabular}

Intervención: Alimentación enteral por sonda

Campo 01.- Fisiológico: básico

Clase D.- Apoyo nutricional

Actividades:
- Observar si hay presencia de sonidos intestinales
cada 4-8 horas, según corresponda.
- Al finalizar la alimentación, esperar 30-60 minutos
antes de colocar al paciente con la cabeza en declive.
- Irrigar la sonda cada 4-6 horas durante la alimentación
continua y después de cada alimentación intermitente.
- Comprobar la existencia de residuos cada 4-6
horas durante las primeras 24 horas y después
cada 8 horas durante la alimentación continua.
- Observar si hay signos de edema o deshidratación.

Fundamentación:

- Los ruidos frecuentes y excesivamente fuertes o

la ausencia de ruidos intestinales podrían indicar

la presencia de una afección subyacente.

- Al finalizar con la nutrición o con la administración de medicación deberá infundir 20-30 ml de agua para evitar obstrucciones de la sonda. Además, se debe mantener hidratado al paciente el aporte hídrico debe realizarse con Sol. fisiológica, aportando las necesidades basales, y la glucosa necesaria para mantener la glucemia entre 100 y $120 \mathrm{mg} / \mathrm{dl}$. Es de suma importancia el control de la natremia y osmolaridad. Tanto la diabetes insípida como la secreción inadecuada de $\mathrm{ADH}$ son complicaciones que pueden empeorar el pronóstico.

\section{Evaluación de proceso}

En la realización de las actividades de enfermería no se presentaron eventos adversos, los cuales complicarían la salud de la paciente. Las actividades se efectuaron de manera satisfactoria.

\section{Evaluación de estructura}

El personal de enfermería involucrado durante la recuperación del paciente contó con las competencias necesarias para la realización de las intervenciones; los recursos materiales fueron los suficientes para poder realizar las mismas.

\section{Evaluación de resultado}

La paciente mostró evidencia de que las intervenciones de enfermería fueron efectivas, realizadas en tiempo y forma.

\section{Plan de cuidados de Enfermería}

Dominio 04: Actividad/ Reposo

Clase 02: Actividad/ Ejercicio

Etiqueta: (00085) Deterioro de movilidad física

Definición: Limitación del movimiento físico independientemente, intencionado del cuerpo o de una o más extremidades.

Diagnóstico de Enfermería: Deterioro de movilidad física r/c deterioro neuromuscular m/p inestabilidad postural, limitación de la capacidad para las habilidades motoras finas y gruesas. 
Clasificación de las Intervenciones de Enfermería

\begin{tabular}{|c|c|c|c|c|}
\hline \multirow[t]{2}{*}{ Resultado } & \multirow[t]{2}{*}{ Indicadores } & \multirow[t]{2}{*}{ Escala de Medición } & \multicolumn{2}{|c|}{ Puntuación Diana } \\
\hline & & & Mantener & Aumentar \\
\hline $\begin{array}{l}\text { Dominio 01: } \\
\text { Salud Funcional } \\
\text { Clase C: Movilidad }\end{array}$ & $\begin{array}{l}20802 \text { Mantenimiento } \\
\text { de la posición corporal }\end{array}$ & $\begin{array}{l}1 \text { Gravemente } \\
\text { comprometido o grave }\end{array}$ & 2 & 4 \\
\hline $\begin{array}{l}\text { Resultado } \\
\text { 0208.- Movilidad }\end{array}$ & 20803 Movimiento muscular & $\begin{array}{l}2 \text { Sustancialmente } \\
\text { comprometido o sustancial }\end{array}$ & 2 & 2 \\
\hline & 20804 Movimiento articular & $\begin{array}{l}3 \text { Moderadamente } \\
\text { comprometido o moderado }\end{array}$ & $\begin{array}{c}3 \\
\mathrm{~T}=7\end{array}$ & $\begin{array}{c}4 \\
\mathrm{~T}=10\end{array}$ \\
\hline $\begin{array}{l}\text { Dominio 01: } \\
\text { Salud Funcional }\end{array}$ & 25501 Alerta disminuida & $\begin{array}{l}4 \text { Levemente } \\
\text { comprometido o levente }\end{array}$ & 3 & 4 \\
\hline $\begin{array}{l}\text { Clase C: Movilidad } \\
\text { Resultado }\end{array}$ & 25502 Estado cognitivo & 5 No comprometido & 3 & 4 \\
\hline $\begin{array}{l}\text { 0205: Consecuencias de la } \\
\text { inmovilidad: Psicocognitivas }\end{array}$ & 25503 Atención disminuida & & $\begin{array}{c}3 \\
T=9\end{array}$ & $\begin{array}{c}4 \\
\mathrm{~T}=12\end{array}$ \\
\hline
\end{tabular}

Intervención: 1800 Ayuda con el autocuidado

Campo 1.- Fisiológico: Básico

Clase F.- Facilitación del autocuidado

Actividades:

- Proporcionar ayuda hasta que el paciente sea totalmente capaz de asumir el autocuidado.

- Ayudar al paciente a aceptar las

necesidades de dependencia.

- Establecer una rutina de actividades de autocuidado.

- Considerar la edad del paciente al promover

las actividades de autocuidado.

- Proporcionar un ambiente terapéutico garantizando una experiencia cálida, relajante, privada y personalizada.
Fundamentación:

- Independientemente de la discapacidad, las personas deben tener intimidad y ser tratadas con dignidad durante las actividades del cuidado personal.

- La limpieza es importante para tener comodidad y una autoestima positiva y para las relaciones sociales con otras personas.

- La discapacidad a menudo genera negación, ira y frustración. Estas son emociones válidas que deben ser identificadas y tratadas.

- Ofrecer opciones e involucrar a la persona en la planificación de su propio cuidado, se disminuyen los sentimientos de impotencia, se promueven los sentimientos de libertad, control y dignidad personal, y se aumenta la disposición del cliente para cumplir los tratamientos.

Intervención: 0844 Cambio de posición: neurológico

Campo 02.- Fisiológico: Complejo

Clase I.- Control Neurológico

Actividades:

- Inmovilizar o apoyar la parte corporal afectada, según corresponda.

- Colocar en la posición terapéutica designada.

- Apoyar la parte corporal afectada.

- Colocar en una cama de flujo de aire, si fuera posible.

- Mantener la alineación corporal correcta.

- Realizar ejercicios pasivos de rango de movimiento en las extremidades afectadas.

- Instruir a los familiares sobre el modo de ayudar al paciente a girarse en la cama y a cómo realizar ejercicios de un modo apropiado.
Fundamentación:

- Esto le ayuda a la piel a mantenerse saludable y prevenir escaras de decúbito.

- Verifique que los tobillos, las rodillas y los codos del paciente no estén descansando uno encima del otro.

- Vigilar la posición correcta de las articulaciones para evitar contracturas, deformidades y rigidez.

- Los cambios de posición contribuyen a conservar el tono muscular y a evitar la fatiga.

- Enseñar al paciente y/o familia las posturas adecuadas que debe adoptar y la forma de conseguirlas.

Intervención: 0740 Cuidados del paciente encamado

Campo 01.- Fisiológico: Básico

Clase C.- Control de la movilidad

Actividades:

- Colocar al paciente con una alineación corporal adecuada.

- Utilizar ropa de cama limpia, seca y sin arrugas.

- Subir barandillas, según corresponda.

- Cambiar de posición al paciente, según lo indique el estado de la piel.

- Vigilar el estado de la piel.

- Aplicar medidas profilácticas antiembólicas.
Fundamentación:

- Valorar el estado del paciente para programar la posición adecuada y la frecuencia de los cambios posturales.

- Extremar las medidas de seguridad durante la movilización del paciente, ante el riesgo de caídas. 


\section{Evaluación de Proceso}

Mientras se llevaron a cabo las intervenciones de enfermería no existió ningún tipo de evento el cual pusiera en riesgo la salud del paciente. Las intervenciones planeadas fueron llevadas a cabo sin problema alguno.

\section{Evaluación de Estructura}

Los profesionales de enfermería involucrados en el cuidado a la paciente postoperada contaban con la experiencia y el conocimiento necesario, por lo cual se pudieron llevar a cabo las intervenciones planeadas.

\section{Evaluación de Resultado}

Las intervenciones realizadas fueron efectivas para la paciente, se realizó de manera minuciosa el cambio de posición, lo que además contribuyó a la prevención de ulceras por presión.

\section{Resultados}

La evaluación del proceso de enfermería arrojó que las intervenciones fueron efectivas. Por un lado, se produjo mejor saturación en la paciente, pasó de $91 \%$ a 97\%, con una frecuencia cardiaca dentro de parámetros normales y coloración de tegumentos adecuada. Si bien, es un tanto complicado restaurar el deterioro de la deglución por el daño de los pares craneales que tiene el paciente, sin embargo, se puede compensar la alimentación, a través de la alimentación por la sonda de gastrostomía con técnica de residuo. Paulatinamente, la paciente podrá recuperar la movilización física, es necesario que se considere mayor rehabilitación a largo plazo; pese a ello, se puede contribuir a la disminución del riesgo de las ulceras por presión, ya que la paciente se encuentra vulnerable a esta situación que compromete su salud.

\section{Conclusiones}

Existe evidencia de diversos planes de cuidado de enfermería, no obstante, enfocados al EVC secundario a ruptura de aneurisma, no se encuentran publicaciones en los últimos cinco años, sólo se identificaron trabajos terminales de grado.

El EVC secundario a ruptura de aneurisma cerebral es una situación grave y devastadora, tanto para el sistema sanitario como para la familia. Lo anterior derivado de la estancia prolongada en las unidades hospitalarias, aunado a los altos costos para la atención de esta patología. Por ello, el PCE en este tipo de pacientes permite identificar los patrones de enfermería alterados y las necesidades frecuentes en el proceso salud-enfermedad del individuo, con el uso de las taxonomías NANDA-NIC-NOC.

El presente artículo permite mostrar evidencia del trabajo que realiza el personal de enfermería quirúrgico, quien además de estar adscrito en el área de quirófano, también puede realizar actividades en el cuidado de los pacientes hospitalizados en los servicios de cirugía. Se muestra la relevancia del cuidado de enfermería, a través de los resultados que muestra el paciente que ha sido sometido a clipaje de aneurisma por ruptura.

\section{Responsabilidades éticas}

Protección de personas y animales. Los autores declaran que para esta investigación no se han realizado experimentos en personas o animales.

Confidencialidad de los datos. Los autores declaran que se aseguró la confidencialidad en el manejo de la información y el consentimiento informado está en resguardo con los autores.

Financiamiento. Estudio autofinanciado por los investigadores.

Conflicto de intereses. Ninguno.

\section{Referencias}

1. Doussoulin A, Rivas R, Sabelle C. Egresos hospitalarios por enfermedad cerebrovascular en el período 
2001-2010 en el Servicio de Salud Araucanía Sur. Rev. méd. Chile 2016; 144(5): 571-6. http://dx.doi.org/10.4067/S0034-98872016000500003

2. Instituto Nacional de Estadística y Geografía, INEGI. Defunciones generales totales por principales causas de mortalidad, 2008. México: INEGI; 2009.

3. Secretaria de Salud. Principales causas de mortalidad general, 2008. México: SINAIS; 2010. [Consultado junio 10 2017]. Disponible en: https://bit.ly/2jz6GXV

4. Pérez-Pupo RD, Leyva-Rojas KM. Mortalidad por enfermedades cerebrovasculares. Hospital Vladimir Ilich Lenin. Correo Científico Médico de Holguín. 2010; 14(2). [Consultado noviembre 25 2017]. Disponible en: https://bit.ly/2HiP7s6

5. Martínez-Barandalla C. Ictus: incidencia, factores de riesgo y repercusión. [Tesis]. España: Universidad Pública de Navarra; 2014. [Consultado octubre 20 2017]. Disponible en: https://bit.ly/2CuSNkZ

6. Instituto Nacional de Neurología y Neurocirugía. Folletos Científicos y Técnicos No. 1 Manual de Normas Técnicas y Lineamientos para la Prevención y Control de las Enfermedades Neurológicas en el Primer Nivel de Atención. México: INNN; 2013: 65-7.

7. Secretaria de Salud-Dirección General de Información en Salud. Boletínes de Información Estadística. México: SSA-DGIS; 2016. [Consultado octubre 20 2017]. Disponible en:https://bit.ly/2RTvHyI

8. Robbins S, Cochran R, Kumar V. Pathological Basis of Disease, $7^{\mathrm{a}}$ ed. Filadelfia: WB Saunders Company; 2010.

9. Venes D. Taber's Cyclopedic Medical Dictionary. 17ª ed. USA: F.A. Davis Company; 1993.

10. Claiborne W, Ridner M. El Manual de Terapéutica Médica, 7ª ed. México: SALVAT; 1993. p.574-9.

11. Mejía CJ, Niño de MC, Ferrer ZL, Cohen MD. Vasoespasmo cerebral secundario a hemorragia subaracnoidea por ruptura de aneurisma intracerebral. Rev. colomb. anestesiol. 2007; 35(2): 143 62. [Consultado septiembre 22 2017]. Disponible en: https://bit.ly/2JrXIVM

12. Argüeso-García M. Pronóstico de la hemorragia subaracnoidea espontánea aneurismática y su correlación con escalas de gravedad, escalas de calidad de vida y tratamiento endovascular. [Tesis doctoral]. España: Universidad de Valencia; 2015.

13. Piloto-González R, Herrera-Miranda GL, Ramos-Águila Y, Mujica-González DB, Gutiérrez-Pérez M. Caracterización clínica-epidemiológica de la enfermedad cerebrovascular en el adulto mayor. Rev Ciencias Médicas 2015; 19(6): 996-1005. [Consultado octubre 19 2017]. Disponible en: https://bit.ly/2ASGiQ6

14. Vives-Medina OT, Quintana-Pereda R, Soto-Páez N. Protocolo de actuación de Enfermería para pacientes con enfermedad cerebrovascular. Rev Ciencias Médicas 2014; 18(3): 414-29. [Consultado octubre 4 2017]. Disponible en: https://bit.ly/2CFbnpq

15. León-Román CA. Aplicación del método cubano de registro clínico del proceso de atención de enfermería. [Tesis doctoral]. La Habana: Instituto de Medicina Tropical Carlos Kourí; 2008. [Consultado mayo 4 2017]. Disponible en: https://bit.ly/2FA2XVe

16. Ángeles-Ávila G, Maciel-Vilchis AC, Cuevas-Peñaloza M, Díaz-Bernal B, Monroy-Martínez MA. Proceso de enfermería en Hospital de Ginecología y Obstetricia del Estado de México, 2016. Cuba: Convención Tecnosalud Camagüey 2017; 2017. [Consultado octubre 20 2017]. Disponible en: https://bit.ly/2RVxHFX

17. Gómez-Rojas ML, Rodríguez-Díaz BL. Situación de enfermería como herramienta para enseñar el proceso de atención de enfermería. rev.cuid. (Bucaramanga. 2010). 2013; 4(1): 544-9. [Consultado octubre 20 2017]. Disponible en: https://bit.ly/2HlWq24

18. González-Salcedo P, Chaves-Reyes AM. Proceso de atención de enfermería desde la perspectiva docente. Inv Enf. 2009; 11(2): 47-76. [Consultado octubre 20 2017]. Disponible en: https://bit.ly/2f2NEWA

19. Herman TH, Kamitsuru S. (Eds.) NANDA International. Diagnósticos Enfermeros. Definiciones y clasificación 2015-2017. España: Elsevier; 2015. 